\title{
Sensitivity and uncertainty analysis of the Variable Infiltration Capacity model in the upstream of Heihe River basin
}

\section{RUI HE \& BO PANG}

College of Water Sciences, Key Laboratory for Water and Sediment Sciences of Ministry of Education, Beijing Normal University, Beijing, 100875, China

herui@mail.bnu.edu.cn, pb@bnu.edu.cn

\begin{abstract}
The increasing water problems and eco-environmental issues of Heihe River basin have attracted widespread attention. In this research, the VIC (Variable Infiltration Capacity) model was selected to simulate the water cycle of the upstream in Heihe River basin. The GLUE (Generalized Likelihood Uncertainty Estimation) method was used to study the sensitivity of the model parameters and the uncertainty of model outputs. The results showed that the Nash-Sutcliffe efficient coefficient was 0.62 in the calibration period and 0.64 in the validation period. Of the seven elected parameters, Dm (maximum baseflow that can occur from the third soil layer), Ws (fraction of the maximum soil moisture of the third soil layer where non-linear baseflow occurs), and d1 (soil depth of the first soil layer), were very sensitive, especially d1. Observed discharges were almost in the range of the $95 \%$ predicted confidence range.
\end{abstract}

Key words VIC model; Heihe; GLUE; uncertainty analyses; runoff simulation

\section{INTRODUCTION}

Heihe River basin, the second largest inland river basin in northwest China, is a water shortage district. It is facing serious ecological and environmental problems caused by the overexploitation of water resources in the recent half century. Hydrological process simulation in Hei River basin is of great scientific significance and practical value in water resource management under various environments (Dong et al., 2012).

Hydrological models, such as the following: TOPMODEL (Han et al., 2004), SWAT (Li and Xu, 2010), improved PRMS model (Zhou et al., 2008), DTVGM (Xia et al., 2003), WEP-Heihe model (Jia et al., 2006) and HIMS model (Wu et al., 2012), have been applied to the Heihe River basin. However, model results can be highly uncertain because of the input data, model structure and model parameter (Li et al., 2009). Reducing the parameter space and accessing the parameter uncertainty are essential to the decision making process.

The purpose of this paper is to employ the large-scale VIC_3L model in simulating the rainfall-runoff process in the upper reaches of Heihe River basin. The GLUE method is adopted to address the VIC model parameter estimation and uncertainty analysis, so as to explore the applicability of the VIC model and supply quantitative support to the management of water resources and ecological protection in this study area.

\section{STUDY AREA DESCRIPTION}

The Hei River is located in the Gansu corridor and the middle section of the Qilian Mountains $\left(97^{\circ} 05^{\prime}-102^{\circ} 00^{\prime} \mathrm{E}\right.$ and $\left.37^{\circ} 45^{\prime}-42^{\circ} 40^{\prime} \mathrm{N}\right)$. According to the altitude and physical geography characteristics, Heihe River basin is divided into mountainous region in upstream, corridor plain area in midstream and Alxa Plateau region in downstream bounded by the Yingluoxia and Zhengyixia hydrological stations. The study area of this paper is the upstream, whose valley area of $10000 \mathrm{~km}^{2}$. It is the runoff and water catchment area of Heihe River basin, and a typical rainmelt water region, with steep terrain as well as a cold and moist climate. The meteorological and hydrological stations information can be seen in Fig. 1.

\section{METHODS}

\subsection{VIC Model}

VIC Model is a large-scale hydrological model, jointly developed by Washington University, the branch school of California University, Berkeley, and Princeton University. As a typical land 


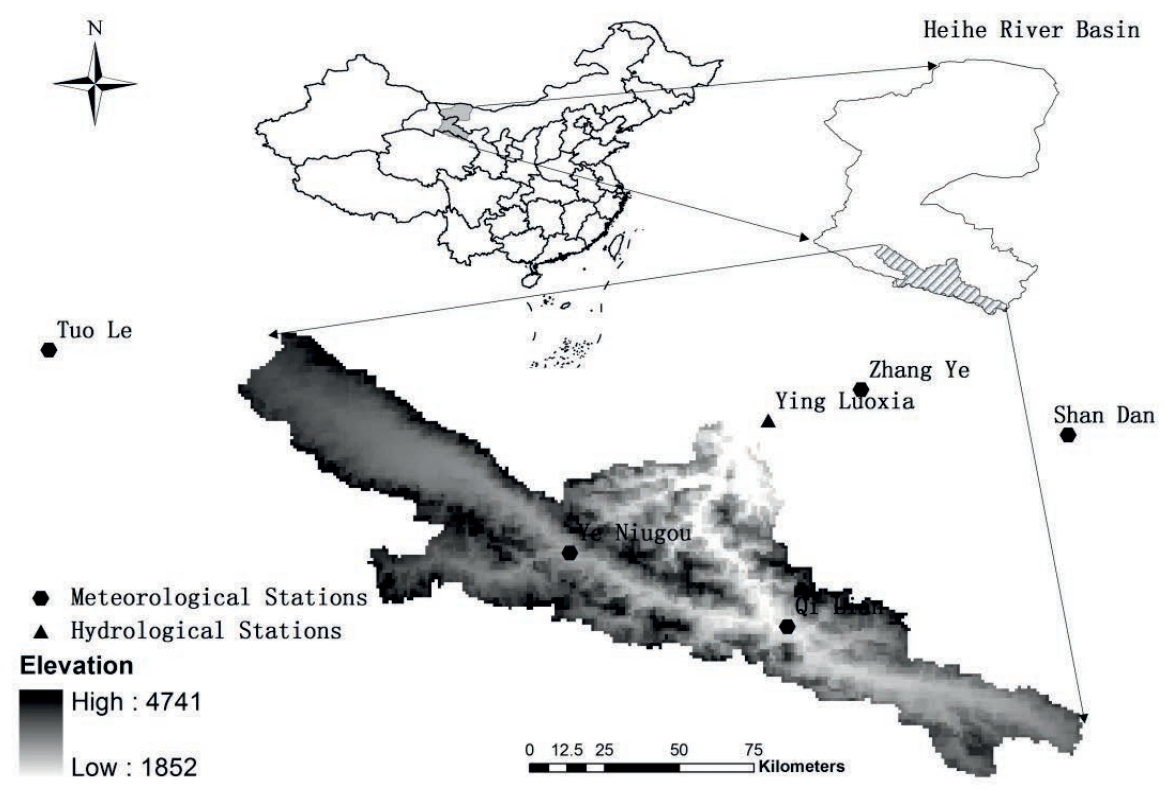

Fig. 1 Map of gauge and meteorological stations and the location of Heihe River basin.

surface hydrological model, the VIC model considers the land surface hydrological processes of infiltration capacity, sub-grid spatial variability and the grid characteristics of parameters, takes into account both the store-full runoff and runoff generation during the infiltration mechanism, and is convenient for nesting with climate model and water resources model to evaluate the impact of climate change on water resources. Since it was developed, the VIC model has been widely used in research on runoff simulation, the effects of climate change on water resources, landatmosphere coupling and the national daily soil moisture simulation; in addition, it has been successfully applied in different scales of basin.

\subsection{GLUE Method}

The GLUE method, proposed by British hydrologists Beven and Binley (Beven and Binley, 1992), is the most representative, early uncertainty analysis method, and can be understood and operated easily. GLUE acknowledges the phenomenon of "equifinality for different parameters" in hydrological simulation and considers that the merits of model simulation results do not depend on a single parameter, but the combination of all parameters (Beven, 2006). The main steps of the GLUE method are as follows (Katri et al., 2006): (1) Monte Carlo sampling from a feasible parameter space with uniform distribution; (2) specification of the likelihood function and the threshold value for behavioural parameter sets; (3) calculation of likelihood values of behavioural parameter sets; (4) rescaling of these values to formulate a cumulative distribution; and (5) derivation of quantiles of uncertainty from the distribution. In this research, the Nash-Sutcliffe efficiency coefficient $\mathrm{E}_{\mathrm{ns}}$ was chosen as the likelihood function, equation (1):

$$
\mathrm{E}_{n s}=1-\frac{\sum_{t=1}^{N}\left(\mathbf{y}_{t}^{o b s}-\mathbf{y}_{t}^{s i m}\right)^{2}}{\sum_{t=1}^{N}\left(\mathbf{y}_{t}^{o b s}-\mathbf{y}_{a v g}^{\operatorname{sim}}\right)^{2}}
$$

where $N$ is the length of the time series, $\mathbf{y}_{t}^{o b s}$ and $\mathbf{y}_{t}^{s i m}$ are respectively the observed and simulated discharge at time $t, \mathbf{y}_{\text {avg }}^{\text {sim }}$ is the mean of observed discharge.

\subsection{Model set-up and data}

The main input of the VIC model includes the soil data set, vegetation data set and forcing data set. The soil data were derived from global 5-min data provided by the NOAA Hydrographic Office. 
The vegetation parameters were based on the global $1-\mathrm{km}$ resolution land-cover data sets developed by the University of Maryland and the Land Data Assimilation System (LDAS) information. The daily series of precipitation, maximum and minimum air temperature from five meteorological stations (Fig. 1) were interpolated into each grid cell using Thiessen polygon method.

The daily observed discharges of Yingluoxia hydrological station are used in model calibration and validation. The available data span from 2001/1/1 to 2008/12/31. In order to reduce uncertainty caused by state values initialization, the data from $2001 / 1 / 1$ to $2002 / 12 / 31$ were used as a warming-up period. Then data from $2003 / 1 / 1$ to $2005 / 12 / 31$ were used for the calibration, and the remaining data, from $2006 / 1 / 1$ to $2008 / 12 / 31$, were used for model validation.

\section{RESULTS AND DISCUSSION}

\subsection{Model simulation and optimum parameters}

The VIC model was used to simulate the daily runoff process and the fitting effects are shown in Fig. 2, where obs and sim stand for observed and simulated values, $P$ represents the average precipitation along the upper reaches of Heihe River Basin (as the average precipitation data was incomplete, data of Yeniugou station located in the centre of this area were used in place of it). Then the objective function values of the calibration period (2003-2005) and validation period (2006-2008) were calculated: in addition to the Nash-Sutcliffe efficiency coefficient $E_{n s}$, root mean square error, RMSE, and mean absolute error, MAE (equations (2) and (3)) were calculated:

$$
\begin{aligned}
& \text { RMSE }=\sqrt{\frac{\sum_{t=1}^{N}\left(\mathbf{y}_{t}^{o b s}-\mathbf{y}_{t}^{s i m}\right)^{2}}{\mathrm{~N}}} \\
& \text { MAE }=\frac{\sum_{t=1}^{N}\left|\mathbf{y}_{t}^{o b s}-\mathbf{y}_{t}^{\text {sim }}\right|}{\mathbf{N}}
\end{aligned}
$$

The $E_{n s}$, RMSE and MAE are 0.62, 29.36 and 20.08 in the calibration period, and 0.64, 35.10 and 23.71 in the validation period. Deviations between the simulated and observed values are small, which indicates that the VIC model can simulate rainfall-runoff processes in the upper reaches of Heihe River Basin successfully.

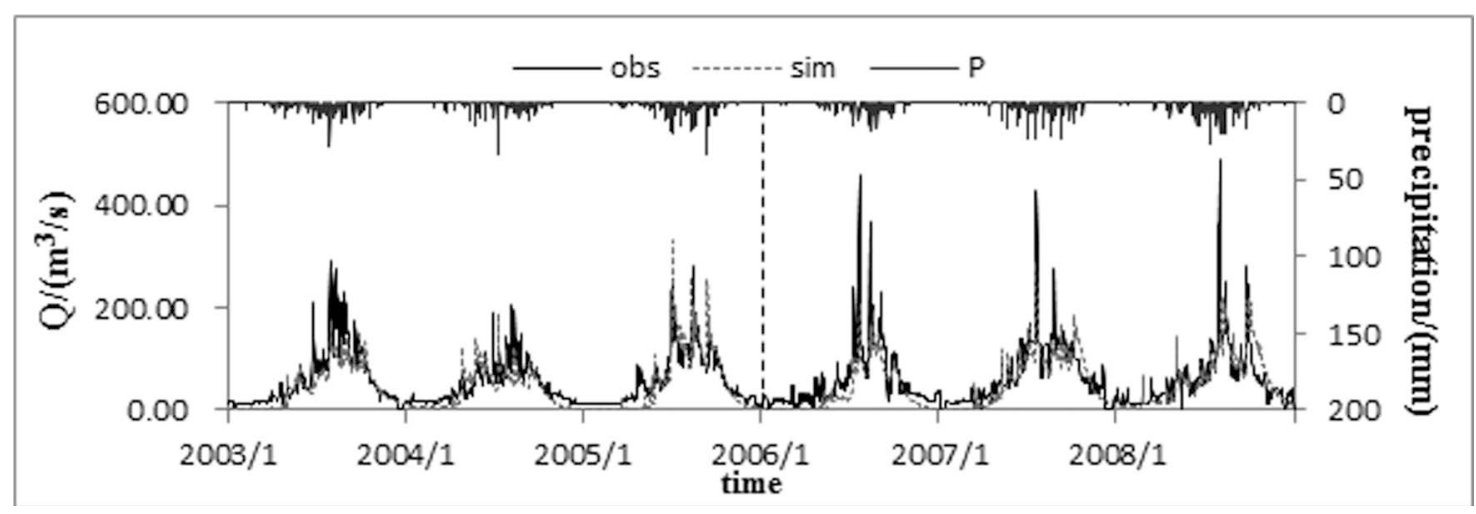

Fig. 2 Simulation results of the VIC model.

\subsection{Sensitivity and uncertainty analysis of model parameters}

The likelihood values of each parameter set were computed and the scatter plot of Nash-Sutcliffe efficiency coefficient $E_{n s}$ drawn against each parameter to analyse the uncertainty of the model parameters (Fig. 3). It can be observed that the parameters are divided into two categories. 

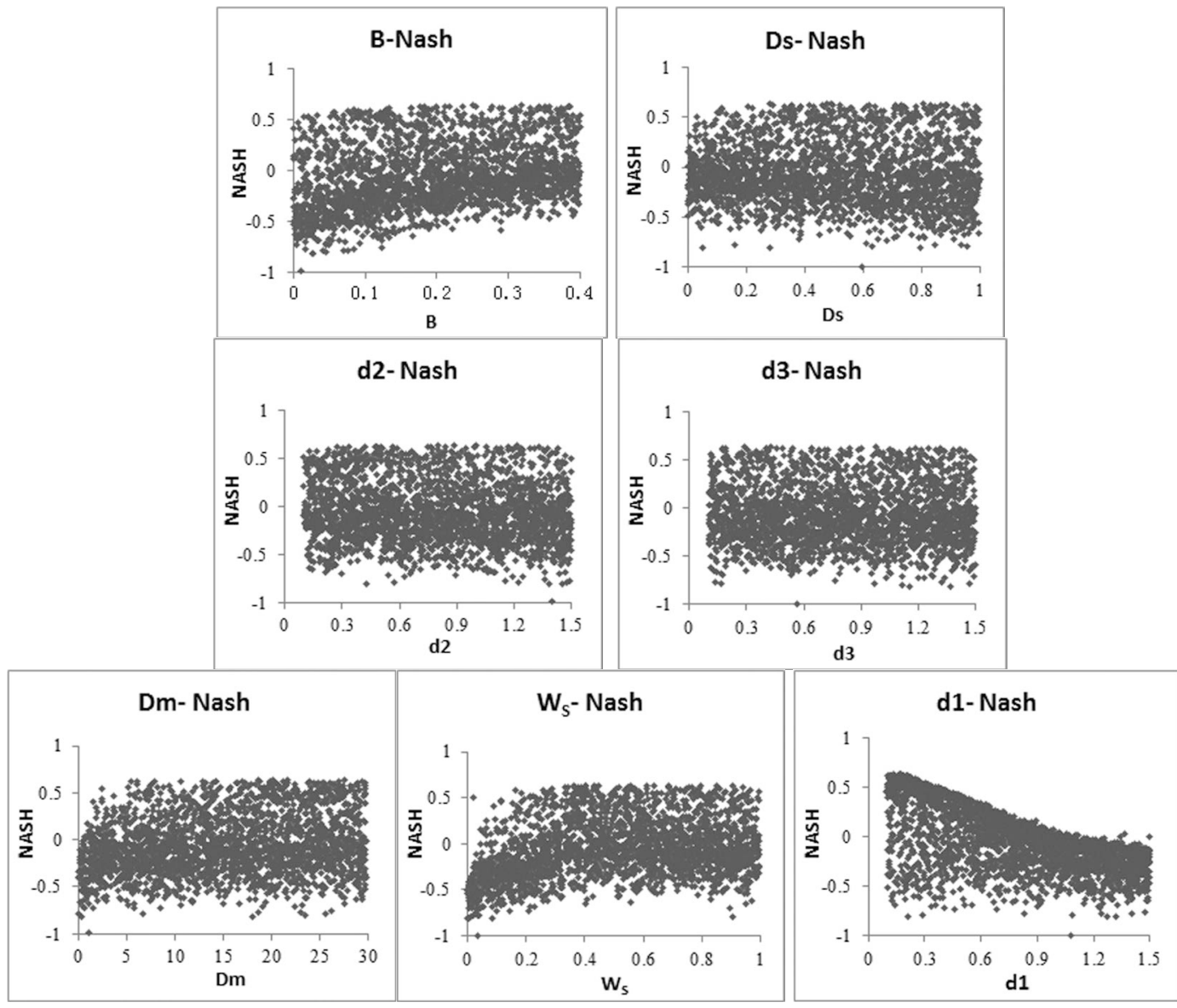

Fig. 3 Scatter plots of likelihood values for VIC parameters.

Insensitive parameters It can be observed that the parameters B (defining the shape of the variable soil moisture capacity curve), Ds (fraction of Dm where non-linear baseflow begins), d2 (soil depth of the second soil layer) and $\mathrm{d} 3$ (soil depth of the third soil layer) are not sensitive. The characteristic of these parameters is that the data scatter evenly and without obvious trend, which demonstrates that the different values of this parameter have less impact on the simulation result. Generally, we deem that the values of Nash-Sutcliffe efficient coefficient are valid when they are greater than zero

Sensitive parameters The changes of parameters $D_{m}, W_{S}$ and $d 1$ have a greater influence on the simulation results. It can be observed that the $E_{n s}$ increases with the rise of Dm (maximum baseflow that can occur from the third soil layer) when the Dm is between 0 and 10, and then remains constant when $\mathrm{Dm}$ is between 10 and 30. It also can be seen that $E_{n s}$ rises when parameter Ws, which is the fraction of maximum soil moisture where non-linear baseflow occurs, rises between 0 and 0.3 and then does not change. In addition, Fig. 3 shows that the diagram of $E_{n s}$ against soil depth of the first soil layer, $\mathrm{d} 1$, not only has a clear peak but also has obvious troughs, indicating the degree of uncertainty of $\mathrm{d} 1$ is remarkable.

\subsection{Uncertainty estimation of discharge simulation}

To calculate the confidence intervals of model uncertainty, a 0.4 threshold was defined, and likelihood values greater than threshold were sorted from largest to smallest and then the models with an uncertainty range under the confidence equal to $95 \%$ obtained. The period from 2005/1/1 to 2007/12/31, for example, is shown in Fig. 4.

The uncertainty range varies with the changes of runoff, being larger at high discharge and less in low flow, so the greatest uncertainty appeared in the peak position, as be seen from the 


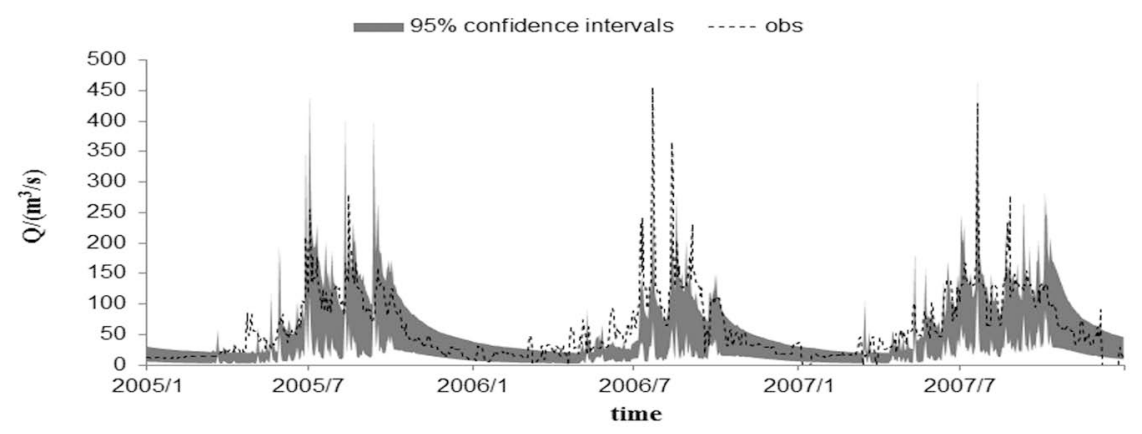

Fig. 4 Uncertainty intervals calculated for runoff event at 95\% confidence level.

diagram. It also can be seen that the actual discharge values are mostly within the $95 \%$ confidence intervals.

\section{CONCLUSION}

This paper simulates the daily runoff process in the upper reaches of Heihe River basin with the VIC model and analyses the model parameter uncertainty using the GLUE method, and then draws the following conclusions:

(1) The VIC model can be used to simulate rainfall-runoff in the upper reaches of Heihe River basin. The $E_{n s}$, RMSE and MAE are separately $0.62,29.36$ and 20.08 in the calibration period, and $0.64,35.10$ and 23.71 in the validation period.

(2) Among the seven parameters of the VIC model, Dm, Ws and d1 are sensitive to the model, and $\mathrm{d} 1$ is the most sensitive. While the remaining parameters (Ds, $\mathrm{B}, \mathrm{d} 2$ and $\mathrm{d} 3$ ) are not sensitive, changes to them have little effect on the model simulation efficiency.

(3) Most of the observations fall within the $95 \%$ confidence intervals; the uncertainty ranges are larger at high discharge and less at low flow.

Acknowledgements This research is supported by two research programs: (1) National Natural Science Foundation of China (Grant No. 51309009). (2) National Natural Science Foundation of China (Grant No. 91125015)

\section{REFERENCES}

Beven, K. and Binley, A. (1992) The future of distributed models-model calibration and uncertainty prediction. Hydrological Processes 6(3), 279-298.

Beven, K. (2006) A manifesto for the equifinality thesis. Journal of Hydrology 320(1-2), 18-36.

Dong, L.H., et al. (2012) Research advances in effects of climate change and human activities on hydrology. Advances in Water Science 23(2), 278-285.

Han, J., Zhang, W.C. and Zhao, D.Z. (2004) TOPMODEL-based simulation of water resources in Heihe River Catchment. Rural Eco-Environment 20(2), 16-20.

Jia, Y.W., Wang, H. and Yan, D.H. (2006) Distributed model of hydrological cycle system in Heihe River Basin - Model development and verification. Journal of Hydraulic Engineering 37(5), 534-542.

Jia, Y.W., Wang, H. and Yan, D.H. (2006) Distributed model of hydrological cycle system in Heihe River Basin Applications. Journal of Hydraulic Engineering 37(6), 655-661.

Katri, R., Tuomo, K. and Dan, B. (2006) An application of the GLUE methodology for estimating the parameters of the INCA$\mathrm{N}$ model. Science of the Total Environment 365,123-139.

Li, Z.L. and Xu, Z.X. (2010) Assessment on hydrological models for runoff simulation in the upper reaches of the Heihe River Basin. Journal of Beijing Normal University (Natural Science) 46(3), 344-349.

Li, Z.L., et al. (2009) Parameter estimation and uncertainty analysis of SWAT model in upper reaches of the Heihe River Basin. Hydrological Processes 23, 2744-2753.

Wu, M.Y., Wang, Z.G. and Dang, S.Z. (2012) Simulation and Analysis of Runoff in the Upper Reaches of the Heihe River Basin. Resources Science 34(10), 1913-1921.

Xia, J., et al. (2003) A research on Distributed Time Variant Gain Modeling. Acta Geographica Sinica 58(5), 789-796.

Zhou, J., et al. (2008) An improved precipitation-runoff model based on MMS and its application in the upstream basin of the Heihe River. Journal of Natural Resources 23(4), 724-736. 